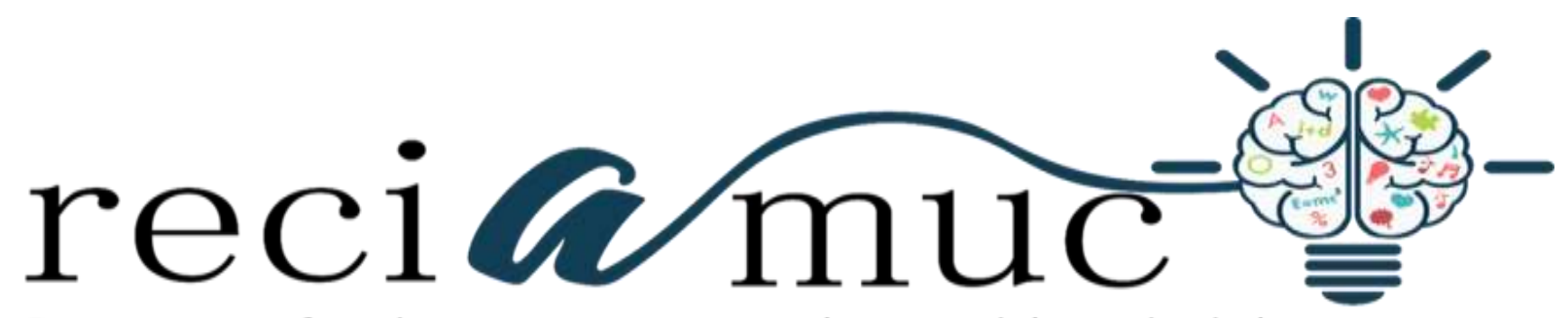

Revista científica de investigación actualización del mundo de las ciencias

Tania Soledad Alvarado Chávez ${ }^{\text {a }}$ : María Antonieta Touriz Bonifaz ${ }^{\text {b; Luis }}$

Francisco Villavicencio Chafla ${ }^{\mathrm{c}}$; Gregory Hernan Pezo Maposa ${ }^{\mathrm{d}}$

Escala funcional de lysholm en los pacientes operados de ligamento cruzado anterior con rigidfix en el Hospital "Teodoro Maldonado Carbo"

Lysholm functional scale in patients operated on anterior cruciate ligament with Rigidfix at the "Teodoro Maldonado Carbo" Hospital

Revista Científica de Investigación actualización del mundo de las Ciencias. Vol. 3 núm., 2, abril, ISSN: 2588-0748, 2018, pp. 32-47

DOI: $10.26820 /$ reciamuc/3.(2).abril.2019.32-47

URL: $\underline{\text { http://reciamuc.com/index.php/RECIAMUC/article/view/325 }}$

Código UNESCO: 3205 Medicina Interna

Tipo de Investigación: Artículo de Investigación

(C) RECIAMUC; Editorial Saberes del Conocimiento, 2019

Recibido: 15/01/2019

Aceptado: 07/02/2019

Publicado: 01/04/2019

Correspondencia: director@reciamuc.com

a. Magister en Educación Superior; Magister en Comunicación y Desarrollo; Especialista en Traumatología y Ortopedia; Diploma Superior en Autoevaluación con Fines de Acreditación; Doctora en Medicina y Cirugía; Universidad de Guayaquil; Guayaquil, Ecuador.

b. Magister en Salud Publica; Magister en Epidemiologia; Medica; Universidad de Guayaquil; Universidad Católica Santiago de Guayaquil; Guayaquil, Ecuador; maria.tourizb@ug.edu.ec - maria.touriz@cu.ucsg.edu.ec

c. Médico; Investigador Independiente; Guayaquil, Ecuador.

d. Médico; Investigador Independiente; Guayaquil, Ecuador. 


\section{Escala funcional de lysholm en los pacientes operados de ligamento cruzado anterior con rigidfix en el Hospital "Teodoro Maldonado Carbo"}

Vol. 3, núm. 2., (2019)

Tania Soledad Alvarado Chávez; María Antonieta Touriz Bonifaz; Luis Francisco Villavicencio

Chafla; Gregory Hernan Pezo Maposa

\section{RESUMEN}

El ligamento cruzado anterior (LCA) de la rodilla se lesiona con mucha frecuencia, la rodilla es una articulación vulnerable a lesiones graves como la inestabilidad anterior. Básicamente existen dos métodos de reparación, con injerto del tendón rotuliano o con injerto de los tendones isquiotibiales. Se determinó la incidencia de inestabilidad anterior crónica del Rodilla, se valoró la evolución postoperatoria y se obtuvieron resultados funcionales con la Escala Lysholm obtenidos en pacientes tratados quirúrgicamente. El presente trabajo es observacional, longitudinal y descriptivo, que incluye a 30 pacientes con inestabilidad anterior de rodilla, en el Hospital Regional IESS Teodoro Maldonado Carbo captados desde el mes de febrero del 2013 hasta el 30 de junio del 2013. Se analizó la información de cada uno de los pacientes mediante un cuestionario tabulado en una hoja de cálculo de Excel. Se realizó la valoración funcional a un total de los pacientes sometidos con a cirugía de reconstrucción del LCA con técnica de RIGIDFIX, a los cuales se les aplico el cuestionario Lysholm, a su vez se dividió la muestra en 2 grupos de pacientes, un grupo comenzó la rehabilitación a los 2 meses y el otro inicio a los 7 días del postoperatorio. Los resultados postoperatorios con la técnica RIGIDFIX son aceptables, con un tiempo de estancia hospitalaria mínima y menor índice de complicaciones.

Palabras Claves: Articulación; Ligamento; Tendón Rotuliano. 


\section{Escala funcional de lysholm en los pacientes operados de ligamento cruzado anterior con rigidfix en el Hospital "Teodoro Maldonado Carbo"}

Vol. 3, núm. 2., (2019)

Tania Soledad Alvarado Chávez; María Antonieta Touriz Bonifaz; Luis Francisco Villavicencio Chafla; Gregory Hernan Pezo Maposa

\section{ABSTRACT}

The anterior cruciate ligament (ACL) of the knee is injured very frequently, the knee is a joint vulnerable to serious injuries such as anterior instability. Basically there are two repair methods, with patellar tendon graft or hamstring graft. The incidence of chronic anterior instability of the Knee was determined, the postoperative evolution was assessed and functional results were obtained with the Lysholm Scale obtained in patients treated surgically. The present work is observational, longitudinal and descriptive, which includes 30 patients with previous instability of knee, at the IESS Teodoro Maldonado Carbo Regional Hospital collected from February 2013 to June 30,2013 . The information of each patient was analyzed using a questionnaire tabulated in an Excel spreadsheet. The functional assessment was performed on a total of patients undergoing ACL reconstruction surgery using the RIGIDFIX technique, to which the Lysholm questionnaire was applied, in turn the sample was divided into 2 groups of patients, one group began the rehabilitation at 2 months and the other start at 7 days post-operative. Postoperative results with the RIGIDFIX technique are acceptable, with a minimum hospital stay time and a lower rate of complications.

Key Words: Joint; Ligament; Patellar Tendon. 


\section{Escala funcional de lysholm en los pacientes operados de ligamento cruzado anterior con rigidfix en el Hospital "Teodoro Maldonado Carbo"}

Vol. 3, núm. 2., (2019)

Tania Soledad Alvarado Chávez; María Antonieta Touriz Bonifaz; Luis Francisco Villavicencio

Chafla; Gregory Hernan Pezo Maposa

\section{Introducción.}

La Inestabilidad Anterior de Rodilla, afecta a hombres adultos jóvenes en etapa productiva, generando un impacto económico-laboral y sobre la funcionalidad del paciente. Por ser una enfermedad que genera un alto grado de incapacidad funcional al no ser tratada oportunamente, es imperativo conocer su incidencia, prevalencia y consecuencias para poder desarrollar protocolos sistemáticos de atención a pacientes con rotura del ligamento cruzado anterior.

La Lysholm Knee Scale es una escala para realizar la valoración funcional de los resultados postoperatorios de la reconstrucción del ligamento cruzado anterior (Castellet, 2010), (Johnson, 2010)

La escala Lysholm es un cuestionario para que sea completado por el propio paciente (Lysholm, 1982), (Tegner, 1985), (Arcuri, 2010), (Lysholm, 2012). Clasifica la satisfacción subjetiva de los pacientes en relación a su capacidad funcional. Consiste en 8 ítems relacionados con la función de la rodilla: cojera, apoyo, subir escaleras, ponerse en cuclillas, inestabilidad, bloqueo, dolor ehinchazón, siendo cada ítem y el puntaje global analizados por separado .Es la más utilizada en la literatura para la evaluar funcionalmente la rodilla en la reconstrucción del ligamento cruzado anterior (Arcuri, 2010), (Risberg, 2009), (Lysholm, 2012). Es un cuestionario que analiza especialmente los síntomas durante las actividades cotidianas y deportivas y tarda pocos minutos en completarse las ocho preguntas. Los resultados se interpretan en pobres si el puntaje está por debajo de 65; regulares si están entre 66 y 83; buenos si son entre 84 hasta 94 y excelentes por encima de 95 puntos (Lysholm, 1982), (Lysholm, 2012). 


\section{Escala funcional de lysholm en los pacientes operados de ligamento cruzado anterior con rigidfix en el Hospital "Teodoro Maldonado Carbo"}

Vol. 3, núm. 2., (2019)

Tania Soledad Alvarado Chávez; María Antonieta Touriz Bonifaz; Luis Francisco Villavicencio Chafla; Gregory Hernan Pezo Maposa

La lesión del ligamento cruzado anterior (LCA) actualmente es la lesión de ligamentos más común de la articulación de la rodilla, se produce en hasta 1 de cada 3.500 personas cada año (Barber-Westin y Noyes, 2011) La pérdida de la función de la LCA causa inestabilidad de la articulación anteromedial de la rodilla. Los cambios en la cinemática de la articulación de la rodilla conduce a un aumento del estrés en los meniscos, con lo cual aumenta el daño al cartílago (Logan et al, 2009). La demanda funcional de la rodilla y el grado de discapacidad importante que deja una rotura del ligamento cruzado anterior crean la necesidad de utilizar métodos fiables de evaluación del tratamiento quirúrgico, para optimizar los resultados y determinar el tipo de cirugía más apropiada para esta lesión.

La inestabilidad anterior de Rodilla, es una lesión frecuente, pero incapacitante, más cuando no se brinda el tratamiento adecuado en el momento oportuno, debido a que si no se realiza una reparación quirúrgica en etapa aguda, puede producir fibrosis de tejido y causar invalidez de la marcha, dificultando las actividades normales del paciente (Bucholz, 2009)

La incidencia de lesiones del LCA depende del deporte y la población estudiada. En una revisión del sistema de Salud de Kaiser, se produjeron lesiones del LCA en el 0,38/1.000 miembros por año, siendo el 67\% de las lesiones relacionadas con el deporte (Paz, 2009).

Muchos Cirujanos ortopédicos cuestionan el valor de los resultados informados por los pacientes por que los consideran de naturaleza subjetiva y menos válida que la objetiva, que se basa en los resultados de las mediciones clínicas. Sin embargo, está comprobado, a partir de diferentes estudios, que la validez de estos cuestionarios referidos por los pacientes son mejores 


\section{Escala funcional de lysholm en los pacientes operados de ligamento cruzado anterior con rigidfix en el Hospital "Teodoro Maldonado Carbo"}

Vol. 3, núm. 2., (2019)

Tania Soledad Alvarado Chávez; María Antonieta Touriz Bonifaz; Luis Francisco Villavicencio

Chafla; Gregory Hernan Pezo Maposa

que los resultados basados solo en datos clínicos objetivos (Risberg, 2009; Lysholm, 2013;

Boden, 2010; Bucholz, 2009; Paz, 2009; Roos, 1998).

La incidencia actualmente es desconocida, aunque hay un aumento de la población de afiliados que consultan a esta institución por lesiones del ligamento cruzado anterior, y siendo la ruptura una lesión incapacitante, con resultados desfavorables si no tiene un tratamiento temprano y adecuado, fundamentan la realización de este estudio.

El objetivo fue realizar una Valoración funcional con la Escala Lysholm a pacientes operados de inestabilidad anterior de rodilla en el Hospital Regional Teodoro Maldonado Carbo, y ofrecer al hospital una forma de evaluación aplicada internacionalmente, que permita obtener los mejores resultados en los pacientes y beneficiar a la sociedad de afilados.

A través de este estudio descriptivo, correlativo y observacional, utilizando la escala Lysholm, que esta validada internacionalmente, aplicada a la información extraída de la historia clínica es posible determinar y caracterizar la población susceptible a un déficit funcional temprano y de esta forma implementar soluciones en su recuperación física. Mediante la determinación de factores de riesgo y presentación de complicaciones se podrá implementar medidas para mejorar los resultados del manejo quirúrgico así como el planteamiento de técnicas que ofrezcan seguridad y mejor estado funcional para los pacientes con Inestabilidad anterior de Rodilla.

Es relevante para la sociedad por que se pretende analizar de forma prospectiva la eficacia, inocuidad, y el costo-beneficio del tratamiento quirúrgico de la inestabilidad anterior de rodilla, a 


\section{Escala funcional de lysholm en los pacientes operados de ligamento cruzado anterior con rigidfix en el Hospital "Teodoro Maldonado Carbo"}

Vol. 3, núm. 2., (2019)

Tania Soledad Alvarado Chávez; María Antonieta Touriz Bonifaz; Luis Francisco Villavicencio Chafla; Gregory Hernan Pezo Maposa

través de la utilización de una escala internacional como es el Lysholm y que sea de utilidad para

el Servicio de Traumatología y Ortopedia ya que no existen escalas para evaluar la ruptura de ligamento cruzado anterior, y de esta manera ofrecer una técnica quirúrgica de simple ejecución, con insumos mínimos y que permite un rápido restablecimiento de los pacientes a sus actividades diarias. Basado en ello, con la incorporación y utilización de una escala de evaluación funcional como la escala Lysholm en el Servicio de traumatología y Ortopedia del Hospital IESS, se pretende depurar y optimizar las técnicas quirúrgicas para obtener los mejores resultados en los pacientes.

En el Hospital Regional $N^{\circ} 2$ Dr. Teodoro Maldonado Carbo hay una incidencia importante de esta lesión con tendencia a incrementarse en el transcurso del tiempo, pero no hay estudios que demuestren información estadística sobre Inestabilidad Anterior de Rodilla. Es interés reportar este estudio en una serie de pacientes y comentar sobre su evolución postoperatoria posterior y paralelamente correlacionar la técnica desarrollada con los resultados funcionales y correlacionar algunos factores de riesgo que puedan motivar esta lesión.

\section{Metodología.}

El universo del estudio incluyó todos los pacientes con Traumatismo severo de rodilla que acuden a consulta externa del Hospital Teodoro Maldonado Carbo. La muestra fue de tipo no probabilística cuantitativa, que tomó en cuenta a los pacientes con diagnóstico de inestabilidad anterior de rodilla, operados en el servicio de Traumatología y ortopedia del Hospital IESS Teodoro Maldonado Carbo desde diciembre 15 hasta el 15 de junio del 2014 


\section{Escala funcional de lysholm en los pacientes operados de ligamento cruzado anterior con rigidfix en el Hospital "Teodoro Maldonado Carbo"}

Vol. 3, núm. 2., (2019)

Tania Soledad Alvarado Chávez; María Antonieta Touriz Bonifaz; Luis Francisco Villavicencio

Chafla; Gregory Hernan Pezo Maposa

La población de estudio debió cumplir con los siguientes criterios de selección:

Se utilizó la observación directa e indirecta, se elaboró una hoja de recolección de datos, la cual se realizó a los pacientes en el momento de ingreso a la unidad hospitalaria. Se utilizó las historias clínicas de los pacientes, las cuales fueron elaboradas por los médicos de postgrado de Traumatología y Ortopedia, donde se detallan antecedentes clínicos, clasificación, diagnóstico, evolución y protocolo operatorio. Posteriormente, ya teniendo seleccionadas las pacientes, se procedió al seguimiento y revisión de historial clínico para obtener los datos pertinentes.

A cada paciente se asignó un número consecutivo mediante orden desde la cirugía realizada con mayor antigüedad hasta la más reciente, no se tuvo en cuenta datos de identificación personal como nombre y documento de identidad, solo se registró el número de historia clínica.

Para el análisis de la información se utilizó una hoja de cálculo de Microsoft Excel y posteriormente analizadas en el programa estadístico IBM SPSS 21. La información se presenta en tablas y gráficos estadísticos para una mejor comprensión, de acuerdo a las variables de estudio. Se utilizó estadística descriptiva, frecuencias absolutas y relativas, además se aplicaron medidas de tendencia central y porcentual.

Para la descripción de las variables se emplearon frecuencias simples, porcentajes, promedios, desviación estándar e intervalos de confianza al 95\%. Para la determinar la relación entre variables cualitativas se empleó la prueba de Chi2 considerándose significativos valores de $\mathrm{P}<0.05$. Para realizar la comparación de las variables se utilizó la prueba U de Mann Whitney. 


\section{Escala funcional de lysholm en los pacientes operados de ligamento cruzado anterior con rigidfix en el Hospital "Teodoro Maldonado Carbo"}

Vol. 3, núm. 2., (2019)

Tania Soledad Alvarado Chávez; María Antonieta Touriz Bonifaz; Luis Francisco Villavicencio Chafla; Gregory Hernan Pezo Maposa

\section{Resultados.}

Se observa que la etiología más frecuente fueron los deportes con 16 casos $(53.33 \%)$ y las caídas con 8 casos (26.66\%), seguidas por los accidentes de tránsito con 6 casos que representan el 20\%. Estos resultados estan acordes con los de la literatura mundial que establecen que las actividades deportivas están relacionadas directamente con las lesiones del LCA entre el 25 al 30\%, que el deporte más frecuente que se asoció a lesión del LCA fue el soccer con el 56\% (9), seguido en menor proporción de atletismo 19\%, voleyball 13\% y basquetball $13 \%$.

El mayor grupo de pacientes con $66.66 \%$ (20 casos) presentaron un giro brusco de la rodilla en valgo/flexión y rotación externa, con el pie fijo en el suelo, seguido de un mecanismo de hiperextensión en varo/flexión y rotación interna con el 13.33\% (4 casos). El 20\% (6 casos) restante no se pudo especificar su mecanismo lesional con exactitud, especialmente en casos de accidente de tránsito y politraumatizados.

Hubo un $26.66 \%$ ( 8 casos) de pacientes con factores de riesgo para lesión del ligamento cruzado anterior y que se relacionaron con los casos de mala evolución clínica en nuestro estudio.

Los factores de riesgo más frecuentes son las enfermedades sistémicas (10\%), seguidas en menor proporción por sedentarismo 7\%, sobrepeso 7\% y las alteraciones anatómicas de rodilla $3 \%$. 


\section{Escala funcional de lysholm en los pacientes operados de ligamento cruzado anterior con rigidfix en el Hospital "Teodoro Maldonado Carbo"}

Vol. 3, núm. 2., (2019)

Tania Soledad Alvarado Chávez; María Antonieta Touriz Bonifaz; Luis Francisco Villavicencio

Chafla; Gregory Hernan Pezo Maposa

El tipo de factores de riesgo encontrado: Hipertensión arterial, Diabetes mellitus y enfermedades de tiroides tuvo una frecuencia de 3.33\% cada uno respectivamente. Además fueron los casos que precisaron interconsultas a especialidades como cardiología y medicina interna para su estabilización y a su vez fueron condicionantes para una mayor estancia hospitalaria.

El $16.66 \%$ de pacientes que se complicaron despues de la cirugía y cuya evolución clínica no fue aceptable, con resultados funcionales regulares.

Las complicaciones más frecuente fueron las sinovitis de rodilla con 3 casos $(9.99 \%)$, seguido de tendinitis de la pata de ganso con 1 casos (3.33\%) y 1 caso con hipotrofia del cuadriceps que representó el (3.33\%).

Tabla 1. Puntaje de funcionalidad pre- postoperatorio con escala Lysholm según sus componentes.

\begin{tabular}{|c|c|c|}
\hline \multicolumn{3}{|c|}{ ESCALA LYSHOLM } \\
\hline No PACIENTES & PREQUIRURGICO & POSTQUIRUGICO \\
\hline 1 & 63 & 90 \\
\hline 2 & 69 & 90 \\
\hline 3 & 68 & 79 \\
\hline 4 & 71 & 90 \\
\hline 5 & 42 & 83 \\
\hline 6 & 69 & 90 \\
\hline 7 & 73 & 90 \\
\hline 8 & 22 & 93 \\
\hline 9 & 47 & 85 \\
\hline 10 & 56 & 85 \\
\hline 11 & 47 & 86 \\
\hline 12 & 67 & 90 \\
\hline
\end{tabular}


Escala funcional de lysholm en los pacientes operados de ligamento cruzado anterior con rigidfix en el Hospital "Teodoro Maldonado Carbo"

Vol. 3, núm. 2., (2019)

Tania Soledad Alvarado Chávez; María Antonieta Touriz Bonifaz; Luis Francisco Villavicencio Chafla; Gregory Hernan Pezo Maposa

\begin{tabular}{|c|c|c|}
\hline 13 & 69 & 90 \\
\hline 14 & 20 & 90 \\
\hline 15 & 74 & 90 \\
\hline 16 & 52 & 85 \\
\hline 17 & 74 & 78 \\
\hline 18 & 47 & 78 \\
\hline 19 & 47 & 78 \\
\hline 20 & 62 & 58 \\
\hline 21 & 35 & 58 \\
\hline 22 & 24 & 58 \\
\hline 23 & 58 & 90 \\
\hline 24 & 76 & 90 \\
\hline 25 & 60 & 90 \\
\hline 26 & 71 & 90 \\
\hline 27 & 76 & 90 \\
\hline 28 & 27 & 83 \\
\hline 29 & 22 & 83 \\
\hline 30 & 22 & 79 \\
\hline PROMEDIO & $\mathbf{5 3 , 6 6}$ & $\mathbf{8 3 , 6 3}$ \\
\hline
\end{tabular}

Fuente: Historia clínica-HRTMC Autor: Investigador

En nuestro estudio se utilizó la escala de Lysholm, para la evaluación funcional de la rodilla antes y después del tratamiento quirúrgico, se establecieron rangos de puntuación: entre 95-100 puntos se considera excelente, buena si esta entre 84-94 puntos, regular si esta entre 6583 puntos y mala si está en el rango menor de 64 puntos. En la tabla 1. Se observó la siguiente distribución de promedios generales:

- Lysholm antes de la cirugía 53,66 puntos, que corresponde a resultados malos.

- Lysholm después de la cirugía 83,63 puntos, que corresponde a resultados regulares.

Los resultados después de la intervención quirúrgica aumentaron significativamente en ambos grupos, observando una mayor puntuación en el grupo de pacientes intervenidos con 


\section{Escala funcional de lysholm en los pacientes operados de ligamento cruzado anterior con rigidfix en el Hospital "Teodoro Maldonado Carbo"}

Vol. 3, núm. 2., (2019)

Tania Soledad Alvarado Chávez; María Antonieta Touriz Bonifaz; Luis Francisco Villavicencio

Chafla; Gregory Hernan Pezo Maposa

técnica $\mathrm{H}-\mathrm{T}-\mathrm{H}$ que presentaron mejores resultados funcionales. Se encontró un valor $\mathrm{p}$ de 0,00012 al comparar los resultados antes y después de la cirugía, demostrando que si existen diferencias significativo entre ambas técnicas quirúrgicas.

Observamos el número y porcentaje de satisfacción preoperatoria y postoperatoria final a los 6 meses de la cirugía, con los siguientes resultados: antes de la cirugía ningún paciente obtuvo resultados excelentes ni buenos, había resultados regulares en 13 casos (43.33\%) y malos en 17 casos (56.66\%). En el postoperatorio final hubieron 19 casos (63.33\%) buenos, 8 casos $(26.66 \%)$ regular y 3 casos malos (10\%). Lo cual indica que obtuvimos un porcentaje de pacientes satisfechos con la cirugía de reconstrucción del LCA con resultados buenos de $63.33 \%$ y un score Lysholm de 89.15 puntos; además se redujo el porcentaje de pacientes insatisfechos antes de la cirugía del $100 \%$ al $36.66 \%$.

Los resultados globales de funcionalidad con la escala Lysholm son los siguientes:

- 3 meses: 53.66 puntos con un grado de satisfacción regular.

- 6 meses: 77.54 puntos con un grado de satisfacción bueno.

- 12 meses: 83.63 puntos con un grado de satisfacción bueno.

Los resultados con la escala Lysholm de los pacientes operados con técnica Rigidfix, encontrando los siguientes resultados:

PREQUIRÚRGICO: Rigidfix de 53.66 puntos. Según la interpretación de la Escala Lysholm un puntaje menor a 64 indica mala función de la rodilla. 


\section{Escala funcional de lysholm en los pacientes operados de ligamento cruzado anterior con rigidfix en el Hospital "Teodoro Maldonado Carbo"}

Vol. 3, núm. 2., (2019)

Tania Soledad Alvarado Chávez; María Antonieta Touriz Bonifaz; Luis Francisco Villavicencio Chafla; Gregory Hernan Pezo Maposa

POSTQUIRÚRGICO: Rigidfix de 83.63 puntos. Según la interpretación de la Escala Lysholm los resultados con RIGIFIX fueron buenos.

Se dividió la muestra de pacientes en dos grupos, el grupo 1 con 15 pacientes, en los cuales la rehabilitación se inició como lo establece la asignación de citas a través de interconsulta por el sistema informático hospitalario AS-400, con un tiempo mínimo de 2 meses; y el grupo 2 con 15 pacientes, en los cuales se introdujo el inicio temprano de la rehabilitación y la marcha precoz a partir de los 7 días postoperatorios.

El grupo 1 de pacientes que realizaron la rehabilitación a los 2 meses de la cirugía y presentaron los siguientes porcentajes de regreso a sus actividades, Actividades ocupacionales: No regresó (3.33\%), regreso con modificaciones de actividades (9.99\%), regreso con limitaciones (6.66\%), regreso completo (30\%). Actividades deportivas: No regresó (13.33\%), regreso con modificación de actividades (16.66\%), regreso con limitaciones (3.33\%) y regreso completo $(16.66 \%)$.

El grupo 2 de pacientes que tuvieron inicio temprano de la rehabilitación (tratamiento integral) obteniendo los siguientes resultados: Actividades ocupacionales: No regresó (0\%), regreso con modificaciones de actividades (3.33\%), regreso con limitaciones $(3.33 \%)$, regreso completo (43.33\%). Actividades deportivas: No regresó (3.33\%), regreso con modificación de actividades $(9.99 \%)$, regreso con limitaciones $(6.66 \%)$ y regreso completo $(30 \%)$.

Evaluamos el índice de satisfacción (escala 1-10) sobre el estado actual de su rodilla intervenida (siendo 0 el peor estado de salud de la rodilla y 10 el mejor). El grado de satisfacción 


\section{Escala funcional de lysholm en los pacientes operados de ligamento cruzado anterior con rigidfix en el Hospital "Teodoro Maldonado Carbo"}

Vol. 3, núm. 2., (2019)

Tania Soledad Alvarado Chávez; María Antonieta Touriz Bonifaz; Luis Francisco Villavicencio

Chafla; Gregory Hernan Pezo Maposa

final de los pacientes fue en el Grupo 1: Muy contentos (16.66\%), contentos (9.99\%) e insatisfechos (23.33\%). En el grupo 2: Muy contentos (30\%), contentos (13.33\%) e insatisfechos (6.66\%). Con un resultado global aceptable de $26.65 \%$ de pacientes que estuvieron satisfechos después de la intervención quirúrgica en el grupo 1 y de $43.33 \%$ en el grupo 2.

Observamos que del total de los 8 pacientes que presentaron factores de riesgo, la mayor parte de ellos tuvo resultados pobres y regulares (7) y solo 1 paciente resultados moderados. Además se observa que si hay relación estadística significativa entre ambas variables, con un valor $\mathrm{p}$ de 0,047 .

Del total de pacientes que tuvieron inicio precoz de rehabilitación (15 casos), todos tuvieron resultados funcionales buenos y moderado con la escala Lysholm, un hubo resultados malos. Además, hubo relación estadística significativa entre ambas variables, con un valor p de 0,0023 .

\section{Conclusiones.}

Al finalizar la investigación y en base a los resultados se concluye que:

Las causas más frecuentes de lesión del LCA fueron las actividades deportivas con el $53.33 \%$, por un mecanismo lesional de valgo forzado con rodilla flexionada y/o en rotación externa $(66,66 \%)$. El factor de riesgo que se presentó en mayor frecuencia fueron las enfermedades sistémicas $10 \%$. 


\section{Escala funcional de lysholm en los pacientes operados de ligamento cruzado anterior con rigidfix en el Hospital "Teodoro Maldonado Carbo"}

Vol. 3, núm. 2., (2019)

Tania Soledad Alvarado Chávez; María Antonieta Touriz Bonifaz; Luis Francisco Villavicencio Chafla; Gregory Hernan Pezo Maposa

Los resultados funcionales con Lysholm antes de la cirugía fueron de 53,66 puntos, que corresponde a resultados malos y Lysholm después de la cirugía de 83,63 puntos, que corresponde a resultados buenos y regulares.

El Grupo 2 dio mejores resultados funcionales y mayores tasas de reincorporación al trabajo y deportes en comparación al grupo 1. Lo cual sugiere que el inicio temprano de la rehabilitación da mejores resultados funcionales y de satisfacción en los pacientes operados de inestabilidad de rodilla.

El inicio temprano de rehabilitación independientemente del protocolo kinésico realizado, favorece los resultados funcionales de la rodilla operada y permite una rápido reincorporación a las actividades deportivas y laborales.

\section{Bibliografía.}

Arcuri F, AbaloE, Barcaly F. Uso de escores para evaluación de resultados en cirugía del ligamento cruzado anterior. ARTROSCOPIA | VOL. 17, No 3: 241-247 | 2010.

Bucholz R., Heckman J. Rockwood \& Green’s Fracturas en el Adulto. Marban. Madrid. Ed. 2009. Pag.1899.

Castellet Feliu E, Vidal N, Conesa X. Escalas de valoración en cirugía ortopédica y traumatología. Trauma Fund MAPFRE (2010) Vol 21 Supl 1: 34-43.

Johnson DS, Smith RB. Outcome measurement in the ACL deficient knee. What's the score? Knee 2010; 8:51-7.

Logan MC, Williams A, Lavelle J, Gedroyc W, Freeman M (2009) Tibiofemoral kinematics following successful anterior cruciate ligament reconstruction using dynamic multiple resonance imaging. Am J Sports Med 32:984- 992. 


\section{Escala funcional de lysholm en los pacientes operados de ligamento cruzado anterior con rigidfix en el Hospital "Teodoro Maldonado Carbo"}

Vol. 3, núm. 2., (2019)

Tania Soledad Alvarado Chávez; María Antonieta Touriz Bonifaz; Luis Francisco Villavicencio

Chafla; Gregory Hernan Pezo Maposa

Lysholm J, Gillquist J. Evaluation of knee ligament surgery results with special emphasis on use of a scoring scale. Am J Sports Med 1982; 10:150-154.

Boden BP, Dean GS Feagin JA et al. Mechanisms of anterior cruciate ligament injury. Orthopedics. 2010;23: 573-578.

Paz C. Plastia de ligamento cruzado anterior con injerto de tendones isquitibiales sin desinserción tibial. Revista de especialidades medico-quirúrgicas, Vol. 14, No 2, abril-junio, 2009, pp 100-102.

Risberg MA, Holm I, Beynnon BD. Sensitivity to changes over time for the IKDC form, the Lysholm score and the Cincinnati Knee score: A prospective study of 120 ACL reconstructed patients with a 2 years follow-up. Knee Surg Sports Traumatol Arthrosc 2009;7: 152-159.

Roos E, Roos H, Lohmander LS, Ekdahl C, Beynnon B. Knee injury and Osteoarthritis Outcome Score (KOOS) - Development of a self-administered outcome measure. The Journal of Orthopaedic and Sports Physical Therapy 78(2)88-96, 1998.

Tegner Y, Lysholm J: Rating systems in the evaluation of knee ligament injuries. Clin Orthop Relat Res 1985; 198:43-49.

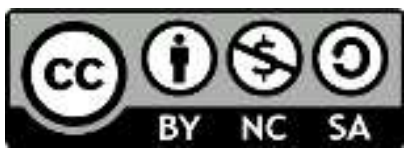

RECONOCIMIENTO-NOCOMERCIAL-COMPARTIRIGUAL

CC BY-NC-SA

ESTA LICENCIA PERMITE A OTROS ENTREMEZCLAR, AJUSTAR Y CONSTRUIR A PARTIR DE SU OBRA CON FINES NO COMERCIALES, SIEMPRE Y CUANDO LE RECONOZCAN LA AUTORÍA Y SUS NUEVAS CREACIONES ESTÉN BAJO UNA LICENCIA CON LOS MISMOS TÉRMINOS. 